Non-equilibrium x-ray spectroscopy using direct quantum dynamics

T. Northey, J. Duffield, and T. J. Penfold

Citation: The Journal of Chemical Physics 149, 124107 (2018); doi: 10.1063/1.5047487

View online: https://doi.org/10.1063/1.5047487

View Table of Contents: http://aip.scitation.org/toc/jcp/149/12

Published by the American Institute of Physics

\section{PHYSICS TODAY}

ADVANCED LIGHT CURE ADHESIVES

READ NOW

$$
\begin{aligned}
& \text { Take a closer look at what these } \\
& \text { environmentally friendly adhesive } \\
& \text { systems can do }
\end{aligned}
$$




\title{
Non-equilibrium x-ray spectroscopy using direct quantum dynamics
}

\author{
T. Northey, J. Duffield, and T. J. Penfold ${ }^{a)}$ \\ Chemistry-School of Natural and Environmental Sciences, Newcastle University, Newcastle upon Tyne \\ NE1 7RU, United Kingdom
}

(Received 7 July 2018; accepted 27 August 2018; published online 26 September 2018)

\begin{abstract}
Advances in experimental methodology aligned with technological developments, such as 3rd generation light sources, X-ray Free Electron Lasers, and High Harmonic Generation, have led to a paradigm shift in the capability of X-ray spectroscopy to deliver high temporal and spectral resolution on an extremely broad range of samples in a wide array of different environments. Importantly, the complex nature and high information content of this class of techniques mean that detailed theoretical studies are often essential to provide a firm link between the spectroscopic observables and the underlying molecular structure and dynamics. In this paper, we present approaches for simulating dynamical processes in X-ray spectroscopy based upon on-the-fly quantum dynamics with a Gaussian basis set. We show that it is possible to provide a fully quantum description of X-ray spectra without the need of precomputing highly multidimensional potential energy surfaces. It is applied to study two different dynamical situations, namely, the core-hole lifetime dynamics of the water monomer and the dissociation of $\mathrm{CF}_{4}^{+}$recently studied using pump-probe X-ray spectroscopy. Our results compare favourably to previous experiments, while reducing the computational effort, providing the scope to apply them to larger systems. https://doi.org/10.1063/1.5047487
\end{abstract}

\section{INTRODUCTION}

$\mathrm{X}$-ray spectroscopies, such as absorption, emission, and inelastic scattering, are powerful tools for probing molecular and electronic structures. With the arrival of femtosecond $\mathrm{X}$-ray sources such as X-ray free-electron lasers (XFELs) ${ }^{1-4}$ and high harmonic generation (HHG), ${ }^{5-9}$ it is becoming increasingly possible to use these techniques to probe timeresolved structural changes on the fundamental time-scales of molecular nuclear motion, i.e., femtoseconds. ${ }^{9-15}$

The high brilliance of these X-ray sources is also making it increasingly possible to perform second-order techniques, such as resonant X-ray emission spectroscopy (RXES), also referred to as resonant inelastic X-ray scattering (RIXS). This can overcome the inherently low resolution of traditional $\mathrm{X}$-ray absorption and emission spectroscopy, which is associated with the very short $(<10 \mathrm{fs})$ lifetime of the core-hole state leading to large lifetime broadening. Indeed, RXES can achieve the spectral resolution $(\sim 50 \mathrm{meV})$ required to go beyond electronic transitions and resolve individual vibronic and vibrational energy levels. ${ }^{16-22}$ Importantly, the resonant nature of RXES means that the intermediate core-hole states involved in the second-order process have a finite lifetime, which have been harnessed to study ultrafast $(<10 \mathrm{fs})$ nuclear $^{20,23,24}$ and electron ${ }^{25-27}$ dynamics. Although such techniques do not offer temporal resolution, they have been successfully used to provide an upper bound of dynamical processes, without the need for specialist equipment.

a)Electronic mail: tom.penfold@ncl.ac.uk
Importantly, regardless of whether the dynamical information is directly sought, i.e., through a pump-probe approach, or intrinsic, as in the case of the core-hole lifetime dynamics of RXES, a time-dependent framework describing the nuclear and/or electron dynamics is essential for achieving a full interpretation of these spectra. For electron dynamics, approaches based upon real time propagation of electronic structure approaches have been used. ${ }^{28}$ In these cases, provided the dynamics of interest are short ( $\leq 3 \mathrm{fs})$, the motion of the nuclei can often be neglected. For nuclear dynamics approaches based upon a fully (electrons and nuclei) quantum description will clearly provide the best approach, but usually suffer from a scaling problem associated with the direct product grid representation. Consequently, they are usually limited to reduced coordinated space models ${ }^{29,30}$ or small molecules for which the full multidimensional potential energy surface can be calculated. ${ }^{31}$ Consequently, a simpler method is to adopt a semiclassical approach and represent the nuclear motion using an ensemble of classical trajectories. ${ }^{17,32,33}$ While these approaches will not exhibit a quantum limit and neglect quantised vibrational motion, they can be successfully used to simulate the experimental signal and scale favourably with system size making them accessible for larger systems, such as the condensed phase. ${ }^{34}$

An alternative approach is to start from the timedependent Schrödinger equation (TDSE) and adopt an expansion of the nuclear wavefunction in terms of Gaussian basis functions (GBFs). This has the potential of achieving a fully quantum description of the time evolution of the TDSE, while retaining, through the use of spatially local GBF, the advantages of the trajectory representation of classical mechanics. ${ }^{35,36}$ Recently a number of 
sophisticated approaches based upon Gaussian basis functions (GBFs) have been developed, including multiple spawning, ${ }^{37,38}$ coupled-coherent states (CCS), ${ }^{39}$ multiconfigurational Ehrenfest (MCE), ${ }^{40}$ the multiple cloning method, ${ }^{41}$ and the direct dynamics (DD) variational multi-configurational Gaussian (vMCG) method. ${ }^{42,43}$ Most importantly, the trajectory characteristics of the GBF in these methods allow them to be implemented within on-the-fly schemes, and so the complicated multidimensional potential energy surface can be calculated as and when it is required, instead of a priori. In the context of X-ray spectroscopy, Neville et al. recently used the $a b$ initio multiple spawning (AIMS) algorithm to study the excited state dynamics of ethylene. ${ }^{44-46}$ They calculated the time-evolving X-ray absorption spectrum during excited state non-adiabatic dynamics, illustrating the potential of femtosecond X-ray spectroscopy to provide new insight into excited state dynamics, especially in the presence of a conical intersection. ${ }^{46}$

In this paper, we implement and test on-the-fly calculation of X-ray spectra of non-equilibrium dynamics using the direct dynamics variational multi-configurational Gaussian (DD-vMCG) method, ${ }^{43}$ which achieves an accurate description of nuclear quantum dynamics by adopting a fully variational solution to the TDSE. Indeed, it should be considered as the trajectory based version of the highly successful Multi-Configurational Time Dependent Hartree (MCTDH) method. ${ }^{47}$ Conceptually similar to the spawning method, DDvMCG adopts the same wavefunction ansatz but retains the coupling between the GBFs and the evolution of the expansion coefficients. The GBFs can therefore be considered as quantum trajectories, in contrast to multiple spawning, whose basis functions follow classical trajectories. We apply this to study two different dynamical situations, namely, the core-hole lifetime dynamics of the water monomer, and the dissociation of $\mathrm{CF}_{4}^{+}$recently studied using pump-probe X-ray spectroscopy. We assess the role of the coupling between the GBFs and the computational expense of the methods. Our results compare favourably to experiments, ${ }^{15}$ while reducing the computational effort, and providing the scope to apply them to larger, more complicated systems.

\section{THEORY AND COMPUTATIONS}

\section{A. Quantum dynamics}

The vMCG method, in comparison to the more widely known MCTDH, provides a trajectory-based representation to solve the TDSE and allows it to be implemented within a direct dynamics (DD) scheme. For a detailed overview, readers are referred to a comprehensive recent review. ${ }^{43}$

Briefly, the vMCG equations of motion (EOMs) are derived from the Dirac-Frenkel variational principle

$$
\left\langle\delta \Psi\left|\hat{H}-i \frac{\partial}{\partial t}\right| \Psi\right\rangle=0,
$$

for which the wavefunction ansatz is a product of timeevolving GBFs and expansion coefficient,

$$
\Psi(\mathbf{x}, t)=\sum_{j=1}^{N} A_{j}(t) g_{j}(\mathbf{x}, t),
$$

where $N$ is the number of GBFs forming the ensemble of trajectories that describes the nuclear wavefunction. For the expansion coefficients, the EOM is written as

$$
i \sum_{l} \dot{A}_{l}=S_{j l}^{-1} \sum_{l}\left(H_{j l}-i \tau_{j l}\right) A_{l}+H_{j l} A_{l} .
$$

Here $H_{j l}$ are elements of the Hamiltonian matrix, $S_{j l}$ are elements of the overlap matrix, and $\tau_{j l}$ are elements of the timederivative matrix. This EOM is the same as the one for the multiple spawning method ${ }^{48}$ and only differs from the standard MCTDH expansion coefficient EOMs by the requirement to address, using $S_{j l}^{-1}$, the non-orthogonality of the GBFs.

The GBFs span the $n$ nuclear degrees of freedom (DOFs) and can be expressed as

$$
g_{j}(\mathbf{x}, t)=\exp \left(\sum_{\kappa}^{n} \varsigma_{j \kappa} x_{\kappa}^{2}+\epsilon_{j \kappa} x_{\kappa}+\eta_{j}\right),
$$

for time-dependent width, linear, and phase parameters, $\varsigma, \epsilon$, and $\eta$, respectively. The EOMs for parameter of GBF can be expressed in matrix notation, ${ }^{4,49-51}$

$$
i \dot{\boldsymbol{\Lambda}}=\boldsymbol{X}+\boldsymbol{C}^{-1} \boldsymbol{Y}
$$

where $\boldsymbol{\Lambda}=\left\{\varsigma_{j}, \epsilon_{j}, \eta_{j}\right\}$. The $\boldsymbol{C}$ matrix contains the GBF derivatives with respect to their coefficients, density terms $A_{j}^{*} A_{l}$, and overlap terms $\left\langle g_{j} \mid g_{l}\right\rangle$. Drawing the connection between vMCG and other GBF approaches, such as multiple spawning, Eq. (5), which is derived by using the local harmonic approximation, separates the propagation of the Gaussian parameters into uncorrelated (classical) and correlated (quantum) terms. ${ }^{43}$ The classical motion of the system is contained within $\boldsymbol{X}$, and the second, correlated part, $\boldsymbol{C}^{-1} \boldsymbol{Y}$, contains all nonclassical effects and the coupling between the Gaussian functions, responsible for the quantum effects such as tunneling. ${ }^{52}$ In this work, truncating the EOM's to only $\boldsymbol{X}$ is denoted as clMCG, which is analogous to multiple spawning with the exception that the basis set size does not vary with time. Quantum GBFs use the full EOMs in Eq. (5) and they are referred to as vMCG.

The total calculation cost of DD-vMCG ${ }^{43,51}$ simulations can be approximated as

$$
t \sim a N+b N^{2}+c N^{3}
$$

This includes: the quantum chemistry calculations, which scale linearly with the number of GBFs, $N$. The classical part of the quantum dynamics scales approximately with $(N \times n)^{2}$ for $n$ degrees of freedom, due to the $(N \times n)^{2}$ evaluations of $\left\langle\dot{g}_{j}|\hat{H}| g_{l}\right\rangle$. The quantum coupling between GBFs is calculated via inversion of the matrix, $\boldsymbol{C}$, and as matrix inversion has cubic computational scaling, this scales with approximately $(N \times n)^{3}$. For simplicity and the reason that for a fixed system $n$ is constant, the $n^{2}$ and $n^{3}$ terms are hidden within the linear scaling coefficients, i.e., $b=b\left(n^{2}\right)$ and $c=c\left(n^{3}\right)$.

\section{B. X-ray spectroscopy}

$\mathrm{X}$-ray absorption spectroscopy, in the usual transmission scheme, ${ }^{10}$ records the changes on a pulse-to-pulse basis with the X-ray transmission through a sample described by the 
Lambert-Beer law. It can be simulated using Fermi's Golden Rule, the first term in Eq. (7). By contrast, RXES, a secondorder spectroscopy requires treatment using the KramersHeisenberg equation, the second term in the following equation:

$$
\begin{aligned}
F(\Omega, \omega)= & \sum_{f}\left|\left\langle f\left|\hat{H}_{i n t}\right| i\right\rangle+\sum_{n} \frac{\left\langle f\left|\hat{H}_{i n t}\right| n\right\rangle\left\langle n\left|\hat{H}_{i n t}\right| i\right\rangle}{E_{i}-E_{n}-\hbar \Omega-i \frac{\Gamma_{n}}{2}}\right|^{2} \\
& \times \frac{\Gamma_{f} / 2 \pi}{\left(E_{i}-E_{f}+\hbar \Omega-\hbar \omega\right)^{2}+\Gamma_{f}^{2} / 4},
\end{aligned}
$$

where $\hbar \Omega$ and $\hbar \omega$ are the energies of the incident and emitted photons, respectively, and $\mathrm{E}_{i}, \mathrm{E}_{n}$, and $\mathrm{E}_{f}$ are the energies of the initial, intermediate, and final states. $\Gamma_{n}$ and $\Gamma_{f}$ are the lifetime broadening associated with the intermediate and final states.

To describe the effect of nuclear dynamics on X-ray spectra, which is the focus of the present work, we adopt two approaches. The first discretises the wavefunction and calculates the spectrum at the centre of each GBF. This is most appropriate if required to calculate a large spectral range, including many excited states. In the second case, we calculate the spectrum directly from the Fourier transform of the autocorrelation function. This approach, common for the optical absorption spectrum using quantum dynamics, is most suitable when only a few states are involved. These are described in detail below.

\section{Sum of Gaussians approach}

Previous methods for calculating non-equilibrium $\mathrm{X}$-ray spectra have post-processed quantum dynamics simulations. ${ }^{29,30}$ In these cases, the X-ray spectrum of the nonstationary wavepacket is calculated as the weighted sum of spectra calculated at each grid point used to describe the nuclear wavepacket. The weighting corresponds to the magnitude of the nuclear wavepacket at that grid point. Unfortunately, this approach scales exponentially with the number of degrees of freedom and therefore simulating an X-ray spectrum including the effect of more than 3-4 nuclear degrees of freedom is intractable.

Similar to the approach of Neville et al. using ab initio multiple spawning (AIMS), ${ }^{44}$ in the present work, using DD-vMCG, the weighted sum does not occur at grid points but at the centre of each individual GBF, computed on-the-fly,

$$
W(\omega, t)=\sum_{j=1}^{N} A_{j}(t) W_{j}\left(\omega ; \mathbf{x}_{j}(t)\right) .
$$

The spectrum $W_{j}\left(\omega ; \mathbf{x}_{j}(t)\right)$ for Gaussian $j$ consists of Lorentzian broadened oscillator strengths, $I\left(\omega_{k}\right)$, for the core excitations at frequency eigenvalues $\omega_{k}$ and depends parametrically on the central coordinates $\mathbf{x}_{j}(t)$ of the $j$ th GBF,

$$
W_{j}\left(\omega ; \mathbf{x}_{j}(t)\right)=\sum_{k} \mathcal{L}\left(\omega-\omega_{k}\right) I\left(\omega_{k}\right),
$$

where $k$ sums over all possible eigenvalues within a chosen energy range. The Lorentzian functions, $\mathcal{L}\left(\omega-\omega_{k}\right)$, are centred at $\omega_{k}$ and have width chosen based on the core hole lifetime of the absorption edge. Importantly, the spectra can be calculated using a range of electronic structure methods and can include the many excited states usually required to compute XAS and XES spectra, although it does neglect the effects of all quantised vibrational degrees of freedom. Indeed, the quantum effects captured within spectra simulated in this way are those reflected in the amplitude of the $\mathrm{GBF}, A_{j}(t)$.

In the present work, the sum of Gaussians approach was used to calculate the carbon K-edge of the dissociation of $\mathrm{CF}_{4}^{+}$following ionisation. Each spectrum was calculated using the core-valence separation algebraic diagrammatic construction (CVS-ADC) $)^{53}$ and $6-311 \mathrm{~g}^{*}$ basis set as implemented in the Q-Chem Package, ${ }^{54}$ with 20 core-valence excitations from the carbon $1 s$ orbital. This was calculated every 2 fs for the first 100 fs of dynamics. Each transition was broadened with a Lorentzian function with a full width half maximum of $1 \mathrm{eV}$. This is significantly greater than the intrinsic corehole lifetime broadening of $77 \pm 6 \mathrm{meV}^{55}$ and therefore also includes some aspects of the instrument resolution reported in Ref. 15.

\section{Autocorrelation approach}

The previously described sum of Gaussians approach neglects the effects of quantised vibrational degrees of freedom. This usually represents no loss of accuracy, as the core-hole lifetime broadening associated with X-ray spectroscopy prohibits such vibrational resolution, and consequently retaining a description of these quantised vibrational states is unnecessary. However, as described in the Introduction, it is becoming possible, using second order spectroscopies such as RXES, to resolve the vibrational structure. ${ }^{21}$ In these vibrationally resolved RXES experiments, the incoming X-ray photon, which is resonant with a corelevel excitation, projects the ground state nuclear wavefunction onto a core-excited state potential energy surface. This is able to evolve during the femtosecond core-hole lifetime. This propagated wavepacket reaches Franck-Condon overlaps with a multitude of vibrationally excited states before decaying back into the electronic ground state in an elastic process, giving rise to a vibrational progression in a highresolution RXES spectrum. Importantly, the lifetime of the intermediate core-hole excited state $(\tau)$ depends on the initial excitation $^{56}$

$$
\tau=\frac{\hbar}{\left(E_{i}-E_{i n}\right)^{2}+\Gamma_{i}^{2}},
$$

where $E_{i}$ and $\Gamma_{i}$ are the energy and width of the intermediate core-hole state, respectively, and $E_{i n}$ is the energy of the incoming X-rays. Consequently, varying $E_{i n}$, it is possible to vary the lifetime and therefore the vibrational progression enabling one to address and subsequently assign different internal degrees of freedom.

To simulate the vibrational component of the RXES spectra, we adopt an approach based upon the Fourier transform of an autocorrelation function. This approach has been widely used for model surfaces. ${ }^{57,58}$ Here it is combined with on-the-fly dynamics. In this approach, the spectrum is 
calculated

$$
I(\omega) \sim \int_{-\infty}^{\infty} C(t) \exp ^{-i \omega t} \mathrm{~d} t,
$$

where $C(t)$ is the autocorrelation function. Before the autocorrelation function is transformed, it is modified slightly. To reduce artefacts associated with the finite propagation time (temporal truncation) which causes ringing artefacts in the spectrum, due to taking the Fourier transform only over a finite time interval (Gibbs phenomenon), the autocorrelation function is multiplied by $\cos ^{2}(n \pi t / 2 T)$, where $n=1,2, \ldots$ and $T$ denotes the final time plus one time step of the autocorrelation function. The autocorrelation function is calculated as

$$
C(t)=\left\langle\psi_{f}(0) \mid \psi_{f}(t)\right\rangle,
$$

where $\psi_{f}$ is the final state nuclear wavefunction; in the case of vibrationally resolved RXES, this is the ground state wavefunction. At $t=0$, it is the wavefunction from the core-hole excited dynamics projected back onto the ground state surface. This is generated by vertical excitation of the vibrational ground state wavefunction into the lowest corehole excited state, described using the maximum overlap method (MOM), ${ }^{59}$ using DFT orbitals. The total spectrum is a sum of spectra generated in this way with weighting of $\exp (-t / \tau),{ }^{60}$ where $\tau$ is the core-hole lifetime, in this case 3.5 fs corresponding to the oxygen K-edge.

\section{RESULTS AND DISCUSSION}

In Subsections III A and III B, we apply the aforementioned methods to study two different dynamical situations, namely, the core-hole lifetime dynamics of the water monomer and the dissociation of $\mathrm{CF}_{4}^{+}$.

\section{A. Time-resolved X-ray spectroscopy for the dissociation of $\mathrm{CF}_{4}^{+}$}

Pertot et al. ${ }^{15}$ recently developed a table-top highharmonic source to study ultrafast X-ray spectroscopy covering the spectral region of $280-350 \mathrm{eV}$. It was used to study the dissociation of $\mathrm{CF}_{4}^{+}$probed at the carbon $\mathrm{K}$-edge, where the dissociation was triggered by a near-infrared pulse which ionised $\mathrm{CF}_{4}$, producing $\mathrm{CF}_{4}^{+}$. This is unstable in its electronic ground state and dissociates into $\mathrm{CF}_{3}^{+}+\mathrm{F}^{61,62}$ Herein we use direct dynamics within both the cIMCG and vMCG framework to calculate the time-resolved X-ray spectra in full nuclear coordinate space.

Figure 1 shows the carbon $\mathrm{K}$-edge for $\mathrm{CF}_{4}, \mathrm{CF}_{4}^{+}$(at the neutral ground state geometry), and $\mathrm{CF}_{3}^{+}$calculated using the $\mathrm{ADC}(1) / 6-311 \mathrm{~g}^{*}$ level of theory. In the energy range considered, the $\mathrm{CF}_{4}$ spectrum corresponds to a single transition at $306.4 \mathrm{eV}$ arising from the carbon 1s orbital excitations into the unoccupied triply degenerate $5 \mathrm{t}_{2}$ orbitals. Upon ionisation, the main feature is slightly blue shifted as the higher charge of the molecule increases the binding energy of the coreelectrons. Additionally, a second peak arises at $304.4 \mathrm{eV}$ because the triple degeneracy is broken due to the hole created upon ionisation. After dissociation and structural rearrangement, the $\mathrm{CF}_{3}^{+}$fragment reduces the molecular symmetry from $\mathrm{T}_{d}$ to $\mathrm{D}_{3 h}$ symmetry. This leads to a greater splitting of the $5 \mathrm{t}_{2}$

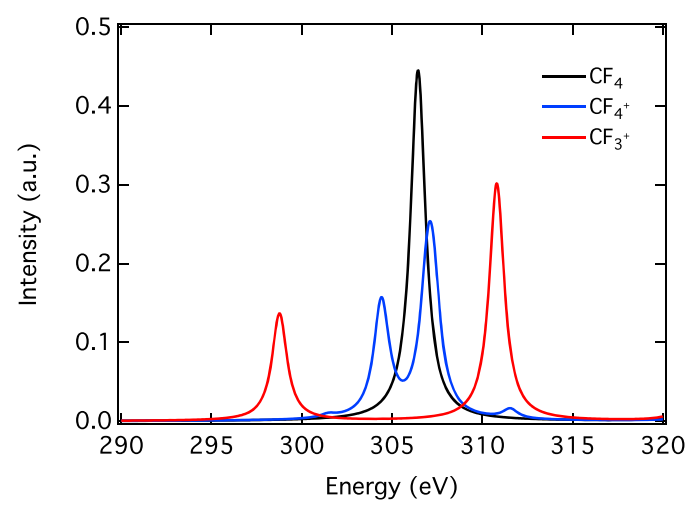

FIG. 1. Carbon $\mathrm{K}$-edge absorption spectra for $\mathrm{CF}_{4}, \mathrm{CF}_{4}^{+}$(at the neutral ground state geometry), and $\mathrm{CF}_{3}^{+}$calculated using the $\mathrm{ADC}(1) / 6-311 \mathrm{~g}^{*}$ level of theory.

orbitals into $5 \mathrm{e}^{\prime}$ and $2 \mathrm{a}_{2}^{\prime \prime}$. This splitting which leads to the main absorption bands has a separation of $\sim 10 \mathrm{eV}$ in good agreement with that reported experimentally in Ref. 15.

The dissociation dynamics were performed using DD-vMCG and DD-clMCG as implemented within the Quantics quantum dynamics package. ${ }^{43}$ In both cases, the initial conditions, which can influence convergence, were a Gaussian function with amplitude 1 centred at the optimised $\mathrm{CF}_{4}$ geometry surrounded by additional basis functions with amplitude 0 with an overlap of 0.6 . They were initiated by projecting the ground state optimised $\mathrm{CH}_{4}$ molecule directly into the lowest ion state. It is noted that the broadband nature of the near-infrared pulse means that experimentally the lowest three ion states are initially populated. However, only the lowest state is dissociative, ${ }^{63,64}$ and the higher lying bound ion states strongly coupled to the lowest states and rapidly undergo internal conversion to the lowest ion state in $<10$ fs. ${ }^{65}$

Figure 2 shows the average $\mathrm{C}-\mathrm{F}$ bond length of the dissociating bond using 10, 20, 40, and 60 GBFs for vMCG (a) and clMCG (b). In both cases, these have been calculated as an average over each GBF weighted by the expansion coefficient, $A_{j}(t)$

$$
r_{C F}(t)=\sum_{j=1}^{N} A_{j}(t) r_{C F, j}(t),
$$
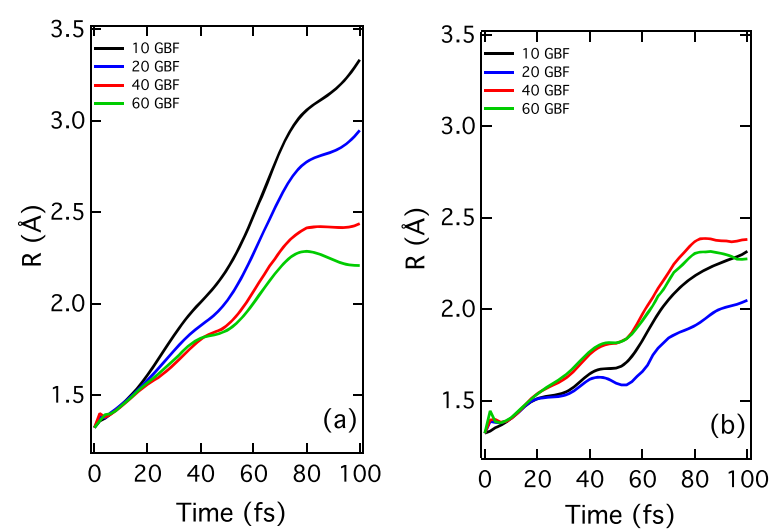

FIG. 2. Weighted average dissociative C-F bond [using Eq. (13)] for 10 (black), 20 (blue), 40 (red), and 60 (green) GBFs using the vMCG (a) and clMCG (b) EOMs. 


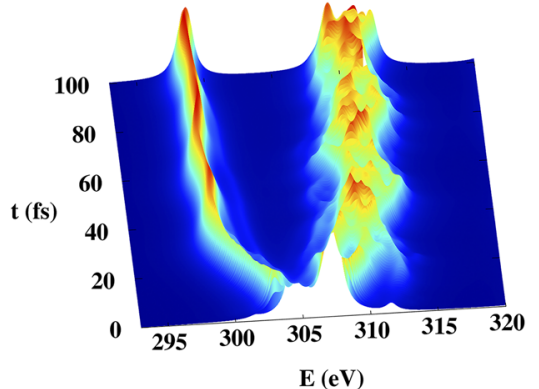

(a)

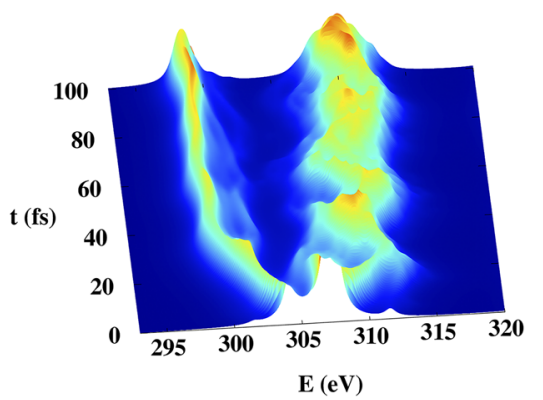

(c)

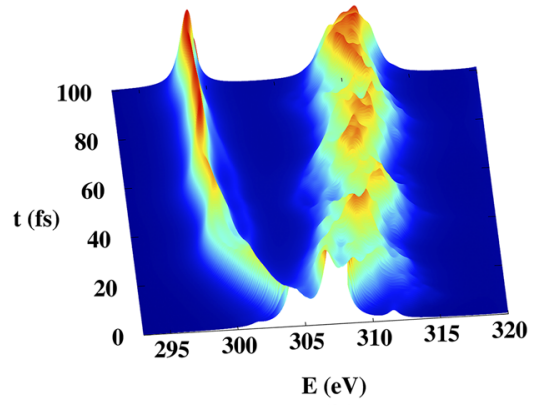

(b)

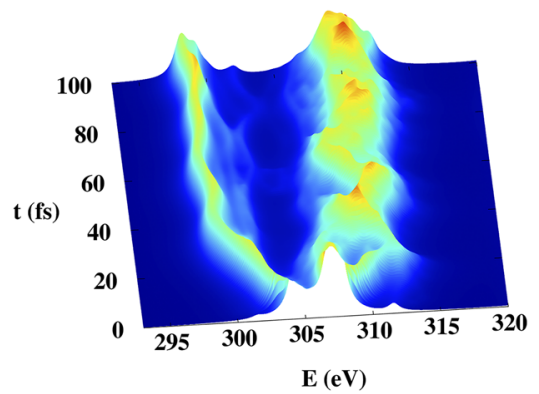

(d)

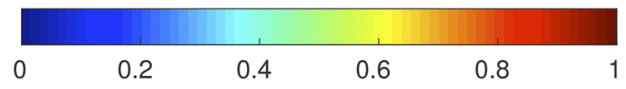

FIG. 3. C K-edge spectra as a function of time calculated from $\mathrm{CF}_{4}^{+}$dissociation simulations using 10 (a), 20 (b), 40 (c), and 60 (d) vMCG GBFs. The colour scale is linear and represents absorption intensity normalised to the largest intensity. where $r_{C F, j}(t)$ are the dissociative $\mathrm{C}-\mathrm{F}$ bond-length for each GBF. Each simulation starts from the ground state vibrational state at equilibrium geometry which has $4 \mathrm{CF}$ bond lengths of
1.32 $\AA$. In all cases, a rapid increase in the $\mathrm{C}-\mathrm{F}$ bond length is observed, indicating dissociation. For both the vMCG and clMCG spectra, there is little difference between the dynamics

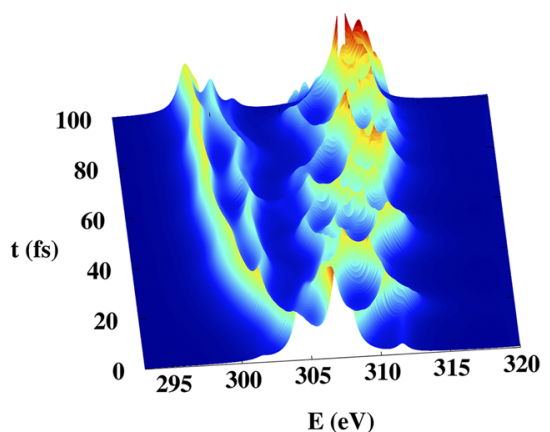

(a)

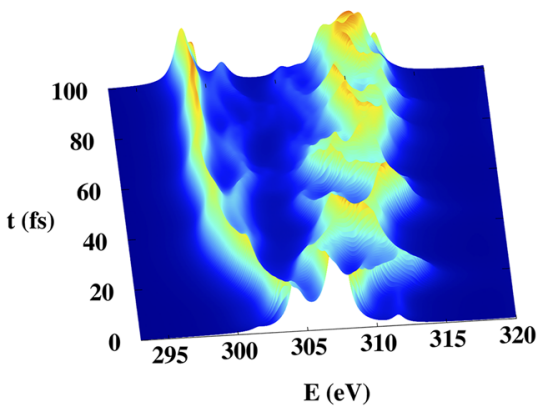

(c)

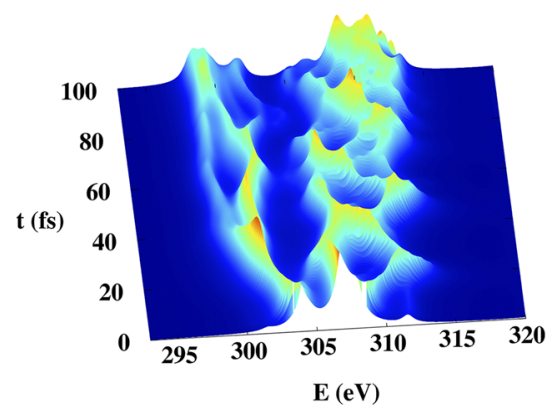

(b)

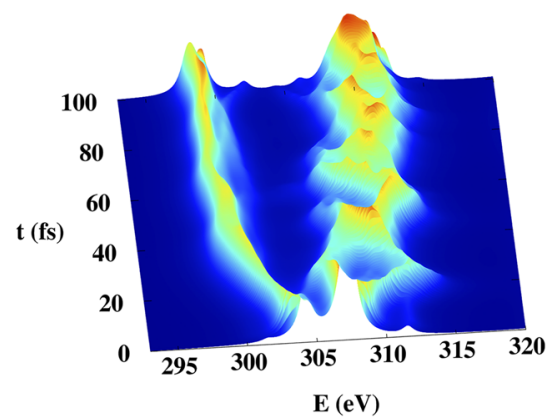

(d)
FIG. 4. C K-edge spectra as a function of time calculated from $\mathrm{CF}_{4}^{+}$dissociation simulations using 10 (a), 20 (b), 40 (c), and 60 (d) clMCG GBFs. The colour scale is linear and represents absorption intensity normalised to the largest intensity. 
simulated using 40 and 60 GBFs indicating reasonable convergence. In addition, the average $\mathrm{C}-\mathrm{F}$ bond length is in good agreement with the $2-2.4 \AA$ reconstructed from the experimental spectra. ${ }^{15}$

It is interesting to note the different convergence observed in $\mathrm{VMCG}$ and clMCG as a function of the number of GBFs. The former (vMCG) shows a clear trend as a function of the number of GBFs and favours faster dissociation for fewer GBFs. This is because the quantum force included within vMCG pushes the GBFs apart meaning that they cover a much larger region of phase space, leading to rapid dissociation. While there remain clear variations, the clMCG trajectories would appear to indicate a smaller change as a function of the number of GBFs. In this case, the simulations with few basis functions favour slightly slower dynamics due to the absence of the quantum force between the GBF and the smaller region of phase space covered.

Figures 3 and 4 show these simulations used to calculate the time-resolved X-ray signal using vMCG and clMCG, respectively. Due to the simplicity of the dynamics, the same qualitative spectral trends are observed in each case. As described from the static spectra in Fig. 1 , at $\mathrm{t}=0, \mathrm{CF}_{4}^{+}$at the $\mathrm{CF}_{4}$ ground state geometry exhibits two main bands and as the dynamics proceed and the structural rearrangement occurs, these split as expected from Fig. 2. However, clear differences are still observed as a function of the number of GBFs, which are now discussed.

For the vMCG simulations (Fig. 3), the spectra show smaller variations as a function of the number of GBFs, despite
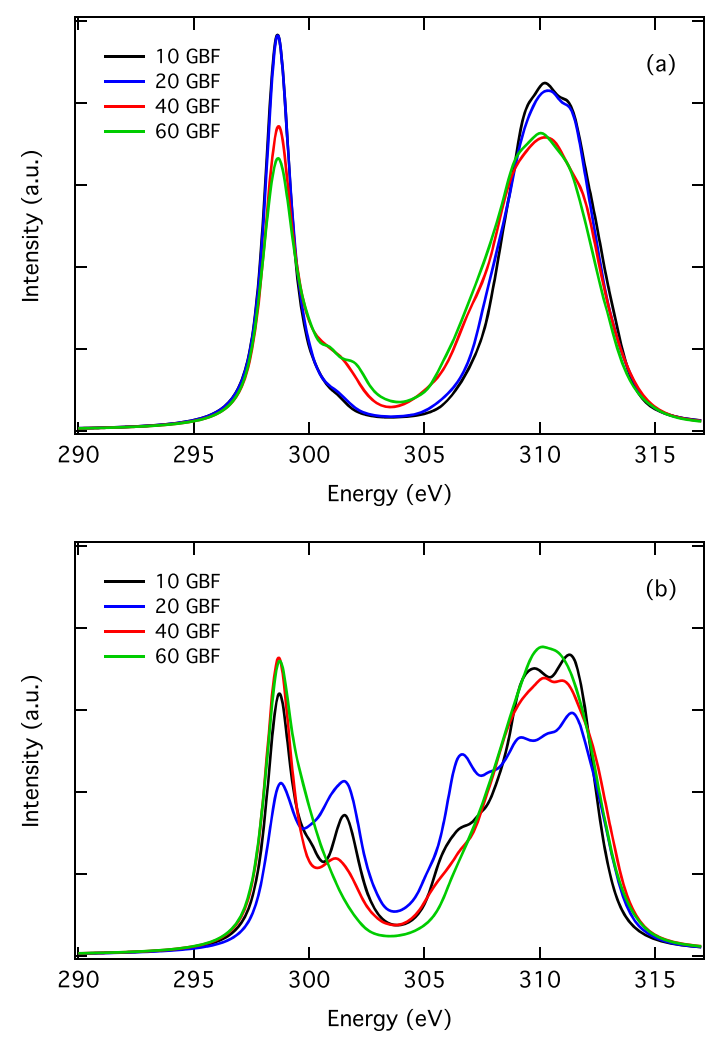

FIG. 5. Temporal integral of the post-dissociation $\mathrm{CF}_{4}^{+}$time-evolving signal, $I(\omega)=\int_{t_{1}}^{t_{2}} W(\omega ; \mathbf{x}(t)) \mathrm{d} t$, between $t_{1}=40$ fs and $t_{2}=100$ fs for $10,20,40$, and 60 GBFs in vMCG (a) and clMCG (b). the apparent larger change in the dissociation dynamics shown in Fig. 2. Indeed, the main change is a slight broadening of the two main bands at longer times, which derives from an increased contribution $\left(A_{j}\right)$ of GBFs which do not completely dissociate within the $100 \mathrm{fs}$ of dynamics. This is consistent with the $\mathrm{C}-\mathrm{F}$ bond lengths shown in Fig. 2(a). This is more clearly seen in Fig. 5, showing the excited state spectrum integrated between 40 and 100 fs. As expected from Fig. 3, the spectra in each case are qualitatively similar with the additional broadening on the high energy side of the lower peak and the lower energy side of the transition at $310 \mathrm{eV}$ only for larger basis sets. Figure 6 shows the rise of the $\mathrm{a}_{2}^{\prime \prime}$ transition at 298.8 $\pm 0.5 \mathrm{eV}$ as a function of time. This shows a principle rise in $\sim 30 \mathrm{fs}$, in reasonable agreement with the $40 \pm 2 \mathrm{fs}$ reported by Pertot et al. ${ }^{15}$

Figure 4 shows the time-evolving $\mathrm{C} \mathrm{K}$-edge spectra calculated using clMCG. Compared to vMCG, larger differences are observed despite the smaller variations in the bond lengths observed in Fig. 2. The temporally integrated signal shown in Fig. 5 reveals a clear doublet structure for a small number of GBFs (10 and 20). This makes the splitting between the two peaks $\sim 3 \mathrm{eV}$, which is in poor agreement with that reported in Ref. 15. As the number of GBFs is increased, the agreement with vMCG increases. The rise of the $\mathrm{a}_{2}^{\prime \prime}$ transition (Fig. 6) also shows a stronger variation as a function of the GBFs, with a rise of $\sim 36$ fs for 60 GBFs.

For the present system, the vMCG approach, as expected, provides faster convergence as a function of the number
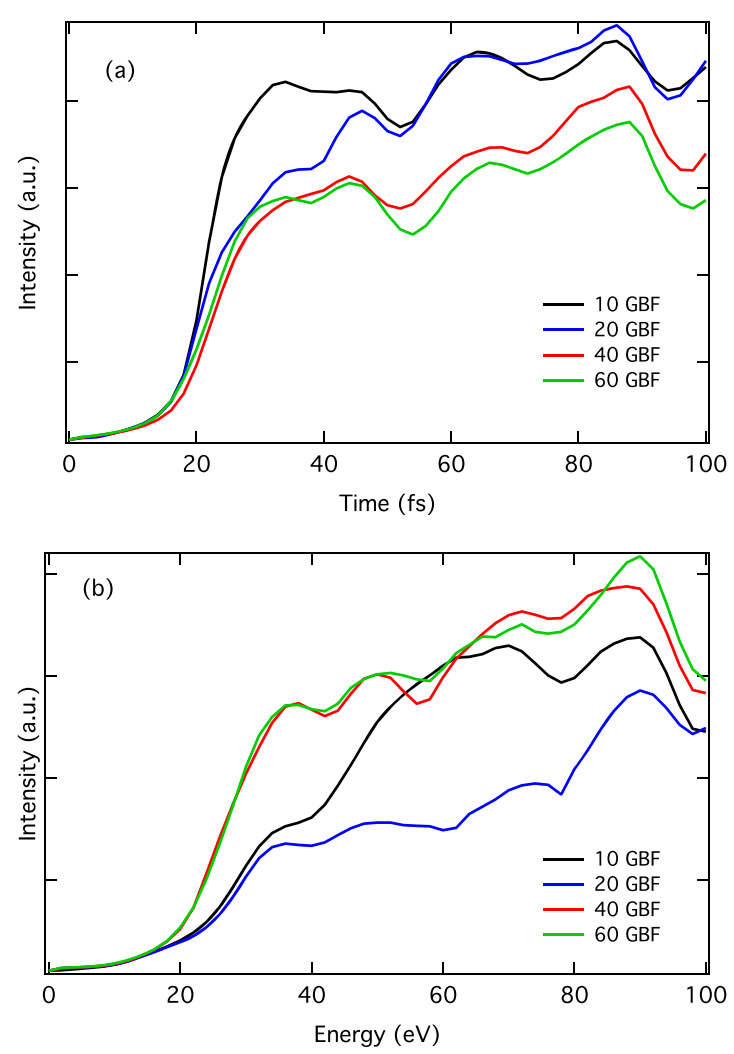

FIG. 6. Time evolution of the X-ray spectra integrated over an energy range of $298.76 \pm 0.48 \mathrm{eV}$ for 10 (black), 20 (blue), 40 (red), and 60 (green) GBFs for vMCG (a) and clMCG (b). 
of GBFs. However, as shown in Eq. (6), this is achieved by incorporating the addition quantum force which scales as $\mathrm{N}^{3}$. Simulating the spectra on-the-fly as performed here also requires additional $d N$ term, where $N$ is the number of GBFs and $d$ is associated with the cost of the quantum chemistry method used to calculate the core-hole spectrum at the centre of each GBF. Table I shows a breakdown for each simulation, using a single thread of an Intel Xeon Processor E5-2620v4 2.10 GHz. The total time for the clMCG dynamics, including the quantum chemistry and the classical GBF propagation, scales roughly linearly with the number of GBFs. This is indicative of a case where the quantum chemistry component (potential and spectra) of the calculations dominates. By contrast, the vMCG scales approximately quadratically, due to the additional computational cost associated with the $C$-matrix inversion. Importantly, despite using a relatively inexpensive method to compute the core-hole excitations, $\mathrm{ADC}(1)$, the quantum chemistry calculations associated with the XAS represents between $30 \%$ and $40 \%$ of the total time. This would be greatly exasperated if a higher level of theory was used such as $\mathrm{ADC}(2)$ or RASPT2 and therefore for these reasons, it is important to minimise the number of GBFs in the simulations. This would appear to favour vMCG.

\section{B. Core-hole dynamics of water}

In RXES, the incoming X-ray photon resonant core-level excitation projects the ground state nuclear wavefunction onto a core-excited state potential energy surface. This intermediate state has an inherent lifetime, i.e., the core-hole lifetime, and before decaying the system is able to propagate during this lifetime. For elastic scattering, the propagated wavepacket on the core-hole excited state reaches Franck-Condon overlap with a multitude of vibrational excited states in the electronic ground state before decaying, giving rise to a vibrational progression in a high-resolution RXES spectrum. ${ }^{21}$ Importantly, by varying the resonant excitation and therefore the lifetime and initial wavepacket in the intermediate state, one can address and subsequently assign specific internal degrees of freedom.

Figure 7 shows the RXES spectrum of a water molecule following excitation into the LUMO $\left(4 a_{1}\right)$. The black line shows the spectrum calculated at the ground state optimised geometry of water, i.e., excluding core-hole lifetime

TABLE I. CPU hours for $\mathrm{CF}_{4}^{+}$DD-clMCG (upper) and DD-vMCG (lower) quantum dynamics simulations and $\mathrm{X}$-ray absorption calculations.

\begin{tabular}{lcccc}
\hline \hline$N$ & $T_{\mathrm{QD}}(\mathrm{h})$ & $T_{\text {XAS }}(\mathrm{h})$ & $T_{\text {total }}(\mathrm{h})$ & $T_{\text {XAS }} / T_{\text {total }}(\%)$ \\
\hline 10 & 5.9 & 4.2 & 10.1 & 41.6 \\
20 & 9.9 & 8.3 & 18.2 & 45.6 \\
40 & 19.4 & 16.7 & 36.1 & 46.3 \\
60 & 27.2 & 25.0 & 52.2 & 47.9 \\
10 & 8.3 & 4.2 & 12.5 & 33.6 \\
20 & 10.7 & 8.3 & 19.0 & 43.7 \\
40 & 26.1 & 16.7 & 42.8 & 39.0 \\
60 & 55.7 & 25.0 & 80.7 & 31.0 \\
\hline \hline
\end{tabular}
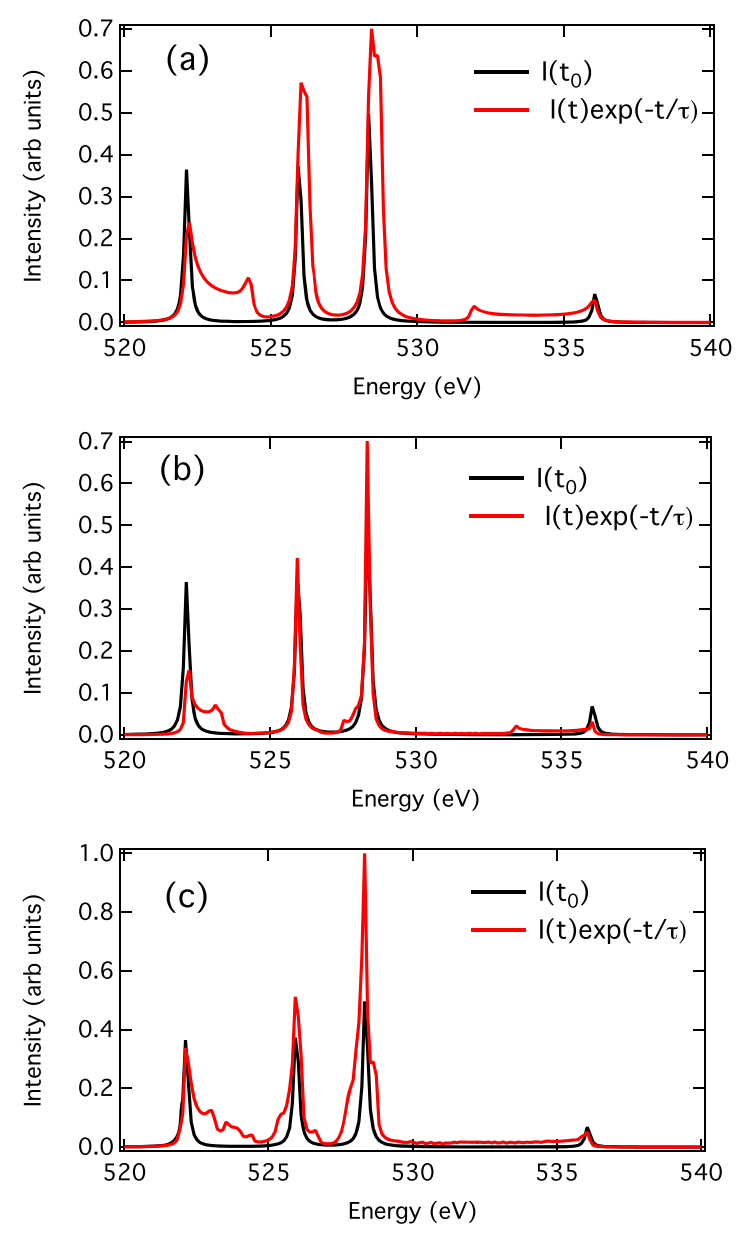

FIG. 7. Simulated RXES spectrum of gas-phase water calculated using a $\operatorname{MOM}(\mathrm{DFT})$-TDDFT approach. The spectrum without any core-hole lifetime dynamics is shown in the black trace and the spectra incorporating the corehole dynamics calculated using DD-vMCG (red trace). (a) $1 \mathrm{GBF}$, (b) $2 \mathrm{GBF}$, and (c) 5 GBF.

dynamics. The red traces show the spectra calculated incorporating the core-hole lifetime dynamics calculated using the MOM(DFT) method ${ }^{66}$ for 1 (a), 2 (b), or 5 (c) GBFs. The short time of the dynamics $(<10 \mathrm{fs})$ means that only a few GBFs are required. The dynamics have been performed using DD-vMCG and the spectrum calculated every $0.1 \mathrm{fs}$. These spectra calculated at the centre of each GBF are weighted by the time-dependent expansion coefficients $\left(A_{j}\right)$ and multiplied by $\exp (-t / \tau)$, where $\tau=3.5$ fs is the lifetime of the oxygen $1 \mathrm{~s}$ core-hole state. All calculated spectra and their weightings are summed to generate the overall spectrum.

The four features in the $\mathrm{I}\left(t_{0}\right)$ spectra (Fig. 7 black trace) correspond to the well documented $1 b_{2}^{-1}, 3 \mathrm{a}_{1}^{-1}, 1 \mathrm{~b}_{1}^{-1}$, and $4 \mathrm{a}_{1}^{-1}$ (from low to high energy) emission transitions. ${ }^{67}$ Upon the inclusion of quantum dynamics, noticeable changes are observed. These are most significant for the $1 b_{2}^{-1}$ and $4 a_{1}^{-1}$ emission. For the former, there is a slight loss of intensity and a large degree of broadening following the dynamics in the $4 \mathrm{a}_{1}$ core-hole state. Similar broadening is also observed for the elastic $\left(4 \mathrm{a}_{1}^{-1}\right)$ scattering line, but towards lower emission energy.

The spectra compare favourably to those reported in Refs. 66 and 67. Only a few GBFs are required to capture 
the effect of the core-hole dynamics, and such simulations have previously been performed using classical trajectories. ${ }^{24}$ In this case, because the resolution cannot resolve quantised vibrational levels, it would appear unnecessary for the majority of the spectrum to use a quantum based approach. Importantly, the peak at $535 \mathrm{eV}$ corresponds to the elastic transition, which is significantly broadened upon the inclusion of core-hole dynamics due to ultrafast core-hole dynamics along the symmetric stretch. However, in contrast to experimental observations, this broadened peak does not exhibit any vibrational fine structure because of the lack of vibrational states account in the sum over Gaussians approach. We therefore adopt the autocorrelation function approach to study the elastic transitions and the vibrational fine structure as described in Sec. II B 2.

Figure 8 shows the vibrationally resolved RXES spectrum calculated for gas phase water following excitation into the $4 \mathrm{a}_{1}$ core-hole intermediate state (1s-LUMO transition). Each spectrum has been calculated using the autocorrelation approach described in Sec. II B 2. Each of the calculated spectra shows good agreement with the experimental spectrum with the main vibrational progression due to the symmetric stretch. ${ }^{68}$ The small peaks visible in between the main progression are due to the asymmetric stretch of the water molecule. The calculated spectra do not show such good agreement with the higher energy loss peaks $(>2.5 \mathrm{eV})$, especially compared to the calculations presented in Ref. 68. However, these calculations were based upon a pre-computed potential energy surface using a high level correlated wavefunction method, which although very accurate limits its applicability to small systems. In the present case, the on-the-fly treatment on a DFT potential means that it can be extended to treat much larger systems, especially

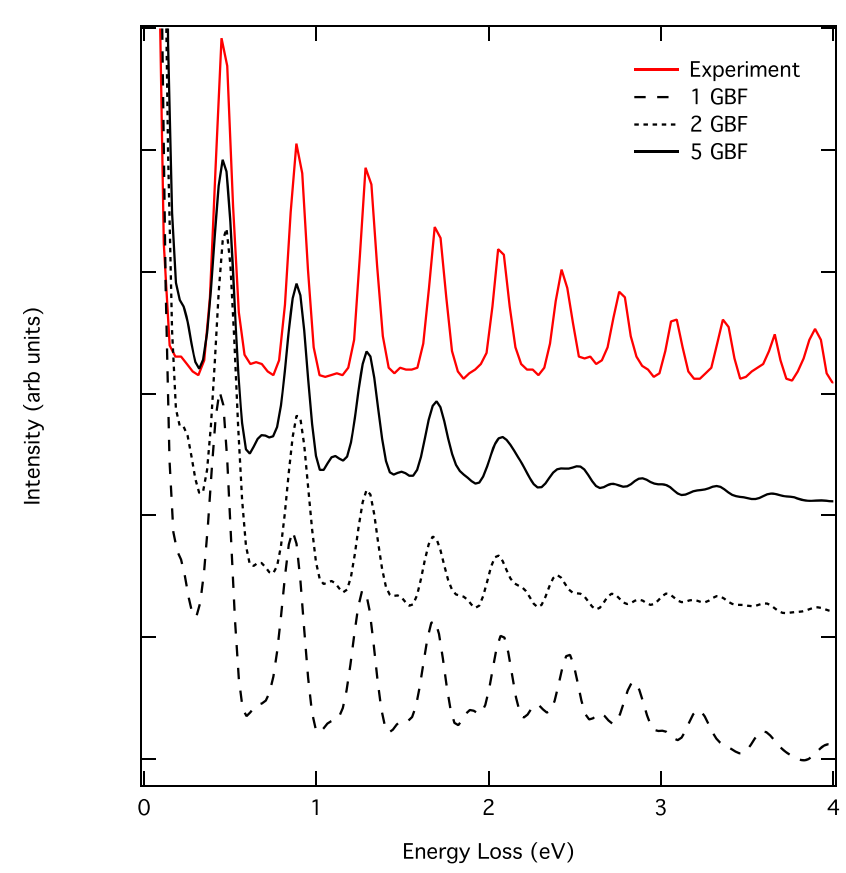

FIG. 8. Vibrational RXES spectrum, plotted as a function of energy loss of gas phase water following excitation into the $4 \mathrm{a}_{1}$ resonance. The experimental spectrum (red) is replotted from Ref. 68. The spectra are calculated with 1 , 2 , and 5 GBFs as indicted inset in the figure. The spectra have been shifted vertically for clarity. as the short time nature of the dynamics reduces the number of GBFs required, and still enable analysis of the experimental spectra.

\section{CONCLUSION}

In this paper, we have presented approaches for simulating the effect of nuclear dynamics on X-ray spectra based upon on-the-fly quantum dynamics with a Gaussian basis set. This was applied to study two different dynamical situations, namely, the core-hole lifetime dynamics of the water monomer and the dissociation of $\mathrm{CF}_{4}^{+}$. The results obtained compare favourably to corresponding experimental data providing new opportunities for first principles interpretation of time-resolved spectra in this manner.

Previous work ${ }^{29,30}$ has highlighted the challenge associated with the exponential scaling of the grid-based approaches for simulating time-resolved X-ray spectra. Importantly, the GBF approach presented here avoids this problem making it possible to incorporate the effect of more than 3-4 nuclear degrees of freedom. Although in this work we have calculated the potential on-the-fly in a direct dynamics format, vMCG can also be used with reduced coordinate model Hamiltonians. Indeed, the localised nature of X-ray spectra and ultrafast dynamics mean that it is often possible to identify a subset of appropriate dimensions to describe the dynamics and time-resolved spectra.

In contrast to the recent work by Neville et al. ${ }^{44,45}$ based upon classically evolving GBF, the vMCG approach adopted here uses a fully variational solution to the TDSE and therefore does not neglect the quantum force existing between the GBFs. It is therefore expected to converge to the quantum solution with fewer GBF. This is somewhat reflected here in the time-resolved spectra of $\mathrm{CF}_{4}^{+}$. Given the computational expense of the ab initio core-valence calculations, this approach would seem appealing, although this will be balanced with the $\mathrm{N}^{3}$ scaling of the quantum correction term shown in Eq. (6). Consequently, it is stressed that the most efficient route to spectral interpretation will be system dependent and no definitive conclusions about this are possible from the present work.

Finally, it is also interesting to note that both here and in Refs. 44 and 45 , the overall spectrum at time $t$ was calculated using the expansion coefficient, $A_{j}(t)$, of the GBF [Eq. (2)] to weight the contribution of each spectrum simulated at the centre of each GBF. An alternative approach adopted to analyze time-resolved scattering experiments used the weighting as a free parameter to fit the experimental spectra. ${ }^{69}$ The present work, which achieves good agreement with experimental data, shows that it is possible to achieve a fully first principles description of the X-ray spectra enabling both the analysis and prediction of experimental signals.

\section{ACKNOWLEDGMENTS}

We acknowledge the Leverhulme Trust, Project No. RPG-2016-103 for funding. 
${ }^{1}$ P. Emma, R. Akre, J. Arthur, R. Bionta, C. Bostedt, J. Bozek, A. Brachmann, P. Bucksbaum, R. Coffee, F.-J. Decker, Y. Ding, D. Dowell, S. Edstrom, A. Fisher, J. Frisch, S. Gilevich, J. Hastings, G. Hays, Ph. Hering, Z. Huang, R. Iverson, H. Loos, M. Messerschmidt, A. Miahnahri, S. Moeller, H.-D. Nuhn, G. Pile, D. Ratner, J. Rzepiela, D. Schultz, T. Smith, P. Stefan, H. Tompkins, J. Turner, J. Welch, W. White, J. Wu, G. Yocky, and J. Galayda, Nat. Photonics 4, 641 (2010).

${ }^{2}$ W. Ackermann, G. Asova, V. Ayvazyan, A. Azima, N. Baboi, J. Bhr, V. Balandin, B. Beutner, A. Brandt, A. Bolzmann, R. Brinkmann, O. I. Brovko, M. Castellano, P. Castro, L. Catani, E. Chiadroni, S. Choroba, A. Cianchi, J. T. Costello, D. Cubaynes, J. Dardis, W. Decking, H. DelsimHashemi, A. Delserieys, G. Di Pirro, M. Dohlus, S. Dsterer, A. Eckhardt, H. T. Edwards, B. Faatz, J. Feldhaus, K. Flttmann, J. Frisch, L. Frhlich, T. Garvey, U. Gensch, Ch. Gerth, M. Grler, N. Golubeva, H.-J. Grabosch, M. Grecki, O. Grimm, K. Hacker, U. Hahn, J. H. Han, K. Honkavaara, T. Hott, M. Hning, Y. Ivanisenko, E. Jaeschke, W. Jalmuzna, T. Jezynski, R. Kammering, V. Katalev, K. Kavanagh, E. T. Kennedy, S. Khodyachykh, K. Klose, V. Kocharyan, M. Krfer, M. Kollewe, W. Koprek, S. Korepanov, D. Kostin, M. Krassilnikov, G. Kube, M. Kuhlmann, C. L. S. Lewis, L. Lilje, T. Limberg, D. Lipka, F. Lhl, H. Luna, M. Luong, M. Martins, M. Meyer, P. Michelato, V. Miltchev, W. D. Mller, L. Monaco, W. F. O. Mller, O. Napieralski, O. Napoly, P. Nicolosi, D. Nlle, T. Nuez, A. Oppelt, C. Pagani, R. Paparella, N. Pchalek, J. Pedregosa-Gutierrez, B. Petersen, B. Petrosyan, G. Petrosyan, L. Petrosyan, J. Pflger, E. Plnjes, L. Poletto, K. Pozniak, E. Prat, D. Proch, P. Pucyk, P. Radcliffe, H. Redlin, K. Rehlich, M. Richter, M. Roehrs, J. Roensch, R. Romaniuk, M. Ross, J. Rossbach, V. Rybnikov, M. Sachwitz, E. L. Saldin, W. Sandner, H. Schlarb, B. Schmidt, M. Schmitz, P. Schmser, J. R. Schneider, E. A. Schneidmiller, S. Schnepp, S. Schreiber, M. Seidel, D. Sertore, A. V. Shabunov, C. Simon, S. Simrock, E. Sombrowski, A. A. Sorokin, P. Spanknebel, R. Spesyvtsev, L. Staykov, B. Steffen, F. Stephan, F. Stulle, H. Thom, K. Tiedtke, M. Tischer, S. Toleikis, R. Treusch, D. Trines, I. Tsakov, E. Vogel, T. Weiland, H. Weise, M. Wellhfer, M. Wendt, I. Will, A. Winter, K. Wittenburg, W. Wurth, P. Yeates, M. V. Yurkov, I. Zagorodnov, and K. Zapfe, Nat. Photonics 1, 336 (2007).

${ }^{3}$ T. Ishikawa, H. Aoyagi, T. Asaka, Y. Asano, N. Azumi, T. Bizen, H. Ego, K. Fukami, T. Fukui, Y. Furukawa, S. Goto, H. Hanaki, T. Hara, T. Hasegawa, T. Hatsui, A. Higashiya, T. Hirono, N. Hosoda, M. Ishii, T. Inagaki, Y. Inubushi, T. Itoga, Y. Joti, M. Kago, T. Kameshima, H. Kimura, Y. Kirihara, A. Kiyomichi, T. Kobayashi, C. Kondo, T. Kudo, H. Maesaka, X. M. Marchal, T. Masuda, S. Matsubara, T. Matsumoto, T. Matsushita, S. Matsui, M. Nagasono, N. Nariyama, H. Ohashi, T. Ohata, T. Ohshima, S. Ono, Y. Otake, C. Saji, T. Sakurai, T. Sato, K. Sawada, K. Seike, K. Shirasawa, T. Sugimoto, S. Suzuki, S. Takahashi, H. Takebe, K. Takeshita, K. Tamasaku, H. Tanaka, R. Tanaka, T. Tanaka, T. Togashi, K. Togawa, A. Tokuhisa, H. Tomizawa, K. Tono, S. Wu, M. Yabashi, M. Yamaga, A. Yamashita, K. Yanagida, C. Zhang, T. Shintake, H. Kitamura, and N. Kumagai, Nat. Photonics 6, 540 (2012).

${ }^{4}$ E. Allaria, R. Appio, L. Badano, W. A. Barletta, S. Bassanese, S. G. Biedron, A. Borga, E. Busetto, D. Castronovo, P. Cinquegrana, S. Cleva, D. Cocco, M. Cornacchia, P. Craievich, I. Cudin, G. D'Auria, M. Dal Forno, M. B. Danailov, R. De Monte, G. De Ninno, P. Delgiusto, A. Demidovich, S. Di Mitri, B. Diviacco, A. Fabris, R. Fabris, W. Fawley, M. Ferianis, E. Ferrari, S. Ferry, L. Froehlich, P. Furlan, G. Gaio, F. Gelmetti, L. Giannessi, M. Giannini, R. Gobessi, R. Ivanov, E. Karantzoulis, M. Lonza, A. Lutman, B. Mahieu, M. Milloch, S. V. Milton, M. Musardo, I. Nikolov, S. Noe, F. Parmigiani, G. Penco, M. Petronio, L. Pivetta, M. Predonzani, F. Rossi, L. Rumiz, A. Salom, C. Scafuri, C. Serpico, P. Sigalotti, S. Spampinati, C. Spezzani, M. Svandrlik, C. Svetina, S. Tazzari, M. Trovo, R. Umer, A. Vascotto, M. Veronese, R. Visintini, M. Zaccaria, D. Zangrando, and M. Zangrando, Nat. Photonics 6, 699 (2012).

${ }^{5}$ T. Popmintchev, M.-C. Chen, D. Popmintchev, P. Arpin, S. Brown, S. Alisauskas, G. Andriukaitis, T. Balciunas, O. D. Mcke, A. Pugzlys, A. Baltuska, B. Shim, S. E. Schrauth, A. Gaeta, C. Hernandez-Garcia, L. Plaja, A. Becker, A. Jaron-Becker, M. M. Murnane, and H. C. Kapteyn, Science 336, 1287 (2012).

${ }^{6}$ E. J. Takahashi, T. Kanai, K. L. Ishikawa, Y. Nabekawa, and K. Midorikawa, Phys. Rev. Lett. 101, 253901 (2008).

${ }^{7}$ A. Ravasio, D. Gauthier, F. R. N. C. Maia, M. Billon, J.-P. Caumes, D. Garzella, M. Gloc, O. Gobert, J.-F. Hergott, A.-M. Pena, H. Perez, B. Carr, E. Bourhis, J. Gierak, A. Madouri, D. Mailly, B. Schiedt, M. Fajardo, J. Gautier, P. Zeitoun, P. H. Bucksbaum, J. Hajdu, and H. Merdji, Phys. Rev. Lett. 103, 028104 (2009).
${ }^{8}$ M.-C. Chen, P. Arpin, T. Popmintchev, M. Gerrity, B. Zhang, M. Seaberg, D. Popmintchev, M. M. Murnane, and H. C. Kaptey, Phys. Rev. Lett. 105, 173901 (2010).

${ }^{9}$ A. R. Attar, A. Bhattacherjee, C. D. Pemmaraju, K. Schnorr, K. D. Closser, D. Prendergast, and S. R. Leone, Science 356, 54 (2017).

${ }^{10}$ T. J. Penfold, C. J. Milne, and M. Chergui, Adv. Chem. Phys. 153, 1 (2013).

${ }^{11}$ C. Milne, T. Penfold, and M. Chergui, Coord. Chem. Rev. 277, 44 (2014).

${ }^{12}$ H. T. Lemke, C. Bressler, L. X. Chen, D. M. Fritz, K. J. Gaffney, A. Galler, W. Gawelda, K. Haldrup, R. W. Hartsock, H. Ihee, J. Kim, K. H. Kim, J. H. Lee, M. M. Nielsen, AB. Stickrath, W. Zhang, D. Zhu, and M. Cammarata, J. Phys. Chem. A 117, 735 (2013).

${ }^{13}$ F. Raksi, K. R. Wilson, Z. Jiang, A. Ikhlef, C. Y. Cote, and J.-C. Kieffer, J. Chem. Phys. 104, 6066 (1996).

${ }^{14}$ A. Bhattacherjee, C. D. Pemmaraju, K. Schnorr, A. R. Attar, and S. R. Leone, J. Am. Chem. Soc. 139, 16576 (2017).

${ }^{15}$ Y. Pertot, C. Schmidt, M. Matthews, A. Chauvet, M. Huppert, V. Svoboda, A. von Conta, A. Tehlar, D. Baykusheva, J. P. Wolf, and H. J. Worner, Science 355, 264 (2017).

${ }^{16}$ F. Hennies, S. Polyutov, I. Minkov, A. Pietzsch, M. Nagasono, F. Gel'mukhanov, L. Triguero, M.-N. Piancastelli, W. Wurth, H. Agren, and A. Fohlisch, Phys. Rev. Lett. 95, 163002 (2005).

${ }^{17}$ M. P. Ljungberg, A. Nilsson, and L. G. M. Pettersson, Phys. Rev. B 82, 245115 (2010).

${ }^{18}$ J.-E. Rubensson, F. Hennies, and A. Pietzsch, J. Electron Spectrosc. Relat. Phenom. 188, 79 (2013).

${ }^{19}$ W. Dong, H. Wang, M. M. Olmstead, J. C. Fettinger, J. Nix, H. Uchiyama, S. Tsutsui, A. Q. R. Baron, E. Dowty, and S. P. Cramer, Inorg. Chem. 52, 6767 (2013).

${ }^{20}$ R. Bohinc, M. Žitnik, K. Bučar, M. Kavčič, L. Journel, R. Guillemin, T. Marchenko, M. Simon, and W. Cao, J. Chem. Phys. 139, 134302 (2013).

${ }^{21}$ A. Pietzsch, Y.-P. Sun, F. Hennies, Z. Rinkevicius, H. O. Karlsson, T. Schmitt, V. N. Strocov, J. Andersson, B. Kennedy, J. Schlappa, A. Fohlisch, J.-E. Rubensson, and F. Gel'mukhanov, Phys. Rev. Lett. 106, 153004 (2011).

${ }^{22}$ F. Hennies, A. Pietzsch, M. Berglund, A. Fohlisch, T. Schmitt, V. Strocov, H. O. Karlsson, J. Andersson, and J.-E. Rubensson, Phys. Rev. Lett. 104, 193002 (2010).

${ }^{23}$ R. Bohinc, M. Žitnik, K. Bučar, and M. Kavčič, J. Chem. Phys. 140, 164304 (2014).

${ }^{24}$ M. Odelius, H. Ogasawara, D. Nordlund, O. Fuchs, L. Weinhardt, F. Maier, E. Umbach, C. Heske, Y. Zubavichus, M. Grunze, J. D. Denlinger, L. G. M. Pettersson, and A. Nilsson, Phys. Rev. Lett. 94, 227401 (2005).

${ }^{25}$ T. Marchenko, S. Carniato, L. Journel, R. Guillemin, E. Kawerk, M. Žitnik, K. Bučar, M. Kavčič, R. Bohinc, M. Petric, V. Vaz da Cruz, F. Gel'mukhanov, and M. Simon, Phys. Rev. X 5, 031021 (2015).

${ }^{26}$ A. Föhlisch, P. Feulner, F. Hennies, A. Fink, D. Menzel, D. Sanchez-Portal, P. M. Echenique, and W. Wurth, Nature 436, 373 (2005).

${ }^{27}$ M. Zharnikov, J. Electron Spectrosc. Relat. Phenom. 200, 160 (2015).

${ }^{28}$ A. Bruner, S. Hernandez, F. Mauger, P. M. Abanador, D. J. LaMaster, M. B. Gaarde, K. J. Schafer, and K. Lopata, J. Phys. Chem. Lett. 8, 3991 (2017).

${ }^{29}$ G. Capano, C. J. Milne, M. Chergui, U. Rothlisberger, I. Tavernelli, and T. J. Penfold, J. Phys. B: At., Mol. Opt. Phys. 48, 214001 (2015).

${ }^{30}$ T. Penfold, M. Pápai, T. Rozgonyi, K. B. Møller, and G. Vankó, Faraday Discuss. 194, 731 (2016).

${ }^{31}$ A. Picón, Phys. Rev. A 95, 023401 (2017).

${ }^{32}$ S. Karsten, S. D. Ivanov, S. G. Aziz, S. I. Bokarev, and O. Kühn, J. Phys. Chem. Lett. 8, 992 (2017).

${ }^{33}$ M. P. Ljungberg, Phys. Rev. B 96, 214302 (2017).

${ }^{34}$ M. Odelius, Phys. Rev. B 79, 144204 (2009).

${ }^{35}$ E. J. Heller, J. Chem. Phys. 62, 1544 (1975).

${ }^{36}$ E. J. Heller, J. Chem. Phys. 75, 2923 (1981).

${ }^{37}$ M. Ben-Nun and T. J. Martınez, J. Chem. Phys. 108, 7244 (1998).

${ }^{38}$ T. J. Martinez, M. Ben-Nun, and R. Levine, J. Phys. Chem. 100, 7884 (1996).

${ }^{39}$ D. V. Shalashilin and M. S. Child, Chem. Phys. 304, 103 (2004).

${ }^{40}$ D. V. Shalashilin, J. Chem. Phys. 130, 244101 (2009).

${ }^{41}$ D. V. Makhov, W. J. Glover, T. J. Martinez, and D. V. Shalashilin, J. Chem. Phys. 141, 054110 (2014).

${ }^{42}$ G. A. Worth and I. Burghardt, Chem. Phys. Lett. 368, 502 (2003).

${ }^{43}$ G. W. Richings, I. Polyak, K. E. Spinlove, G. A. Worth, I. Burghardt, and B. Lasorne, Int. Rev. Phys. Chem. 34, 269 (2015).

${ }^{44}$ S. P. Neville, V. Averbukh, S. Patchkovskii, M. Ruberti, R. Yun, M. Chergui, A. Stolow, and M. S. Schuurman, Faraday Discuss. 194, 117 (2016).

${ }^{45}$ S. P. Neville, V. Averbukh, M. Ruberti, R. Yun, S. Patchkovskii, M. Chergui, A. Stolow, and M. S. Schuurman, J. Chem. Phys. 145, 144307 (2016). 
${ }^{46}$ S. P. Neville, M. Chergui, A. Stolow, and M. S. Schuurman, Phys. Rev. Lett. 120, 243001 (2018).

${ }^{47}$ M. H. Beck, A. Jäckle, G. A. Worth, and H. D. Meyer, Phys. Rep. 324, 1-105 (2000).

${ }^{48}$ B. F. Curchod and T. J. Martínez, Chem. Rev. 118, 3305 (2018).

${ }^{49}$ I. Burghardt, H.-D. Meyer, and L. Cederbaum, J. Chem. Phys. 111, 2927 (1999).

${ }^{50}$ I. Burghardt, K. Giri, and G. A. Worth, J. Chem. Phys. 129, 174104 (2008).

${ }^{51}$ T. Penfold, Phys. Chem. Chem. Phys. 19, 19601 (2017).

${ }^{52}$ K. E. Spinlove, G. Richings, M. A. Robb, and G. Worth, Faraday Discuss. (published online 2018).

${ }^{53}$ J. Wenzel, M. Wormit, and A. Dreuw, J. Comput. Chem. 35, 1900 (2014).

${ }^{54}$ Y. Shao, Z. Gan, E. Epifanovsky, A. T. B. Gilbert, M. Wormit, J. Kussmann, A. W. Lange, A. Behn, J. Deng, X. Feng, D. Ghosh, M. Goldey, P. R. Horn, L. D. Jacobson, I. Kaliman, R. Z. Khaliullin, T. Kuś, A. Landau, J. Liu, E. I. Proynov, Y. M. Rhee, R. M. Richard, M. A. Rohrdanz, R. P. Steele, E. J. Sundstrom, H. L. Woodcock III, P. M. Zimmerman, D. Zuev, D. Albrecht, E. Alguire, B. Austin, G. J. O. Beran, Y. A. Bernard, E. Berquist, K. Brandhorst, K. B. Bravaya, S. T. Brown, D. Casanova, C.-M. Chang, Y. Chen, S. H. Chien, K. D. Closser, D. L. Crittenden, M. Diedenhofen, R. A. DiStasio, Jr., H. Do, A. D. Dutoi, R. G. Edgar, S. Fatehi, L. Fusti-Molnar, A. Ghysels, A. Golubeva-Zadorozhnaya, J. Gomes, M. W. D. Hanson-Heine, P. H. P. Harbach, A. W. Hauser, E. G. Hohenstein, Z. C. Holden, T.-C. Jagau, H. Ji, B. Kaduk, K. Khistyaev, J. Kim, J. Kim, R. A. King, P. Klunzinger, D. Kosenkov, T. Kowalczyk, C. M. Krauter, K. U. Lao, A. D. Laurent, K. V. Lawler, S. V. Levchenko, C. Y. Lin, F. Liu, E. Livshits, R. C. Lochan, A. Luenser, P. Manohar, S. F. Manzer, S.-P. Mao, N. Mardirossian, A. V. Marenich, S. A. Maurer, N. J. Mayhall, E. Neuscamman, C. M. Oana, R. Olivares-Amaya, D. P. O’Neill, J. A. Parkhill, T. M. Perrine, R. Peverati, A. Prociuk, D. R. Rehn, E. Rosta, N. J. Russ, S. M. Sharada, S. Sharma, D. W. Small, A. Sodt, T. Stein, D. Stuck, Y.-C. Su, A. J. W. Thom, T. Tsuchimochi, V. Vanovschi, L. Vogt, O. Vydrov, T. Wang, M. A. Watson, J. Wenzel, A. White, C. F. Williams, J. Yang, S. Yeganeh, S. R. Yost, Z.-Q. You, I. Y. Zhang, X. Zhang, Y. Zhao, B. R. Brooks, G. K. L. Chan, D. M. Chipman, C. J. Cramer, W. A. Goddard III, M. S. Gordon, W. J. Hehre, A. Klamt, H. F. Schaefer III, M. W. Schmidt, C. D. Sherrill, D. G. Truhlar, A. Warshel, X. Xu, A. Aspuru-Guzik, R. Baer, A. T. Bell, N. A. Besley, J.-D. Chai, A. Dreuw, B. D. Dunietz, T. R. Furlani, S. R. Gwaltney, C.-P. Hsu, Y. Jung, J. Kong,
D. S. Lambrecht, W.-Z. Liang, C. Ochsenfeld, V. A. Rassolov, L. V. Slipchenko, J. E. Subotnik, T. Van Voorhis, J. M. Herbert, A. I. Krylov, P. M. W. Gill, and M. Head-Gordon, Mol. Phys. 113, 184 (2015).

${ }^{55}$ T. X. Carroll, K. J. Borve, L. J. Saethre, J. D. Bozek, E. Kukk, J. A. Hahne, and T. D. Thomas, J. Chem. Phys. 116, 10221 (2002).

${ }^{56}$ J. Szlachetko, J. Hoszowska, J.-C. Dousse, M. Nachtegaal, W. Blachucki, Y. Kayser, J. Sa, M. Messerschmidt, S. Boutet, G. J. Williams, C. David, G. Smolentsev, J. A. van Bokhoven, B. D. Patterson, T. J. Penfold, G. Knopp, M. Pajek, C. J. Milne, and R. Abela, Sci. Rep. 6, 33292 (2016).

${ }^{57}$ G. Worth, H.-D. Meyer, and L. Cederbaum, J. Chem. Phys. 105, 4412 (1996).

${ }^{58}$ T. Penfold and G. Worth, J. Chem. Phys. 131, 064303 (2009).

${ }^{59}$ A. T. B. Gilbert, N. A. Besley, and P. M. W. Gill, J. Phys. Chem. A 112, 13164 (2008).

${ }^{60}$ O. Takahashi, M. Odelius, D. Nordlund, A. Nilsson, H. Bluhm, and L. G. Pettersson, J. Chem. Phys. 124, 064307 (2006).

${ }^{61}$ D. M. P. Holland, A. W. Potts, A. B. Trofimov, J. Breidbach, J. Schirmer, R. Feifel, T. Richter, K. Godehusen, M. Martins, A. Tutay, M. Y. M. AlHada, S. Eriksson, and L. Karlsson, Chem. Phys. 308, 43 (2005).

${ }^{62}$ J. C. Creasey, H. M. Jones, D. M. Smith, R. P. Tuckett, P. A. Hatherly, K. Codling, and I. Powis, Chem. Phys. 174, 441 (1993).

${ }^{63}$ H. Tachikawa, J. Phys. B: At., Mol. Opt. Phys. 33, 2367 (2000).

${ }^{64}$ J. G. de la Vega and E. San Fabián, Chem. Phys. 151, 335 (1991)

${ }^{65}$ X. Tang, X. Zhou, Z. Sun, S. Liu, F. Liu, L. Sheng, and B. Yan, J. Chem. Phys. 138, 094306 (2013).

${ }^{66}$ A. E. Fouda, G. I. Purnell, and N. A. Besley, J. Chem. Theory Comput. 14, 2586 (2018).

${ }^{67}$ L. Weinhardt, A. Benkert, F. Meyer, M. Blum, R. G. Wilks, W. Yang, M. Bär, F. Reinert, and C. Heske, J. Chem. Phys. 136, 144311 (2012).

${ }^{68}$ V. Vaz da Cruz, E. Ertan, R. C. Couto, S. Eckert, M. Fondell, M. Dantz, B. Kennedy, T. Schmitt, A. Pietzsch, F. F. Guimaraes, H. Agren, F. Gel'mukhanov, M. Odelius, A. Fhlisch, and V. Kimberg, Phys. Chem. Chem. Phys. 19, 19573 (2017).

${ }^{69}$ M. P. Minitti, J. M. Budarz, A. Kirrander, J. S. Robinson, D. Ratner, T. J. Lane, D. Zhu, J. M. Glownia, M. Kozina, H. T. Lemke, M. Sikorski, Y. Feng, S. Nelson, K. Saita, B. Stankus, T. Northey, J. B. Hastings, and P. M. Weber, Phys. Rev. Lett. 114, 255501 (2015). 